\title{
EQUIVALENT CONDITIONS FOR THE EXISTENCE OF UNCONDITIONAL BASES OF REPRODUCING KERNELS IN SPACES OF ENTIRE FUNCTIONS
}

\begin{abstract}
We consider a reproducing kernel radial Hilbert space of entire functions and prove the equivalence of several sufficient conditions for the existence of unconditional bases of reproducing kernels in such spaces.
\end{abstract}

Key words: Hilbert spaces, entire functions, reproducing kernels, unconditional bases, Riesz bases

2020 Mathematical Subject Classification: 46E22, 30D10

1. Introduction. Let $H$ be a radial functional Hilbert space of entire functions, stable with respect to dividing, i. e.:

1) all evaluation functionals $\delta_{z}: f \rightarrow f(z)$ are continuous;

2) if $F \in H$, then $\|F\|=\left\|F\left(z e^{i \varphi}\right)\right\|$ for any $\varphi \in \mathbb{R}$;

3) if $F \in H, F\left(z_{0}\right)=0$, then $F(z)\left(z-z_{0}\right)^{-1} \in H$.

The first condition means that $H$ is a reproducing kernel Hilbert space. Let $k(\lambda, z)$ be its reproducing kernel at $z \in \mathbb{C}$, that is

$$
f(z)=(f(\lambda), k(\lambda, z)), \quad \forall z \in \mathbb{C}, \quad \forall f \in H .
$$

By $K(z)$ we denote $k(z, z)$. Then the Bergman function of the space $H$ is $\left\|\delta_{z}\right\|_{H}=(K(z))^{\frac{1}{2}}$.

A basis $\left\{e_{k}, k=1,2, \ldots\right\}$ in a Hilbert space is an unconditional basis if there exist numbers $c, C>0$, such that for each element $x=\sum_{k=1}^{\infty} x_{k} e_{k} \in H$, the identity holds:

$$
c \sum_{j=1}^{\infty}\left|c_{k}\right|^{2}\left\|e_{k}\right\|^{2} \leqslant\left\|\sum_{j=1}^{\infty} c_{k} e_{k}\right\|^{2} \leqslant C \sum_{j=1}^{\infty}\left|c_{k}\right|^{2}\left\|e_{k}\right\|^{2} .
$$

(C) Petrozavodsk State University, 2021 
An unconditional basis $\left\{e_{j}, j=1,2, \ldots\right\}$ becomes the Riesz basis if and only if $0<\inf _{k}\left\|e_{k}\right\| \leqslant \sup _{k}\left\|e_{k}\right\|<\infty$. Equivalently, a Riesz basis is a linear isomorphic image of an orthonormal basis in a separable Hilbert space.

The problem of existence and construction of unconditional bases of reproducing kernels $\left\{k\left(\lambda, z_{j}\right)\right\}_{j=1}^{\infty}$ (or Riesz bases of normalized reproducing kernels $\left.\left\{k\left(\lambda, z_{j}\right) /\left\|k\left(\cdot, \lambda_{j}\right)\right\|\right\}_{j=1}^{\infty}\right)$ in Hilbert spaces of analytic functions has been actively studied during the recent years. This problems goes back to closely related classical problems: representation of the functions by exponential series and interpolation by entire functions. Namely, an unconditional basis of exponentials in a Hilbert space is an isomorphic image of a basis of reproducing kernels in some Hilbert space of (entire) functions.

Absence of unconditional bases of reproducing kernels in the classical Bargmann space and Fock-type spaces

$$
\mathcal{F}_{\varphi}=\left\{f \in H(\mathbb{C}):\|f\|^{2}:=\int_{\mathbb{C}}|f(\lambda)|^{2} e^{-2 \varphi(\lambda)} d m(\lambda)<\infty\right\},
$$

with radial weights $\varphi$ growing faster than $|\lambda|^{2}$ was shown in works [1-3], in terms of the interpolation by entire functions. The authors of [4] proved absence of unconditional bases of reproducing kernels in spaces with weights obeying the conditions $\left(\ln _{+} r\right)^{2}=o(\varphi(r)), r \rightarrow \infty$ and possessing some regularity of the growth. In the same work, an unexpected result was obtained: existence of unconditional bases of reproducing kernels in the Fock spaces $\mathcal{F}_{\varphi_{\alpha}}$ with the weights $\varphi_{\alpha}(\lambda)=\left(\ln _{+}|\lambda|\right)^{\alpha}$ as $\alpha \in(1 ; 2]$. Later, in paper [5], existence of unconditional bases of reproducing kernels in the Fock spaces with radial weights of essentially more general form was proved. Note that all these papers deal with Fock spaces with radial weights. As far as the authors know, unconditional bases in non-radial spaces have been considered only in [6-8].

It was proved in [9] (see Theorem 1) that if $H$ is a radial functional Hilbert space stable with respect to dividing, admitting a Riesz basis of normalized reproducing kernels, and monomials are complete in $H$, then there exists a convex sequence $u(n), n \in \mathbb{N} \cup\{0\}$, such that $\|z\|^{n} \asymp e^{u(n)}$, $n \in \mathbb{N} \cup\{0\}$. The convexity of $\{u(n)\}$ means

$$
u(n+1)+u(n-1)-2 u(n) \geqslant 0, \quad n \in \mathbb{N} .
$$

If $u(t)$ is a convex piece-wise linear function with integer nonnegative breakpoints, and $u(t) \equiv u(0)$ as $t<0$, then the convexity condition (1) 
can be written in a more compact form

$$
u_{+}^{\prime}(n+1)-u_{+}^{\prime}(n) \geqslant 0 .
$$

Here $u_{+}^{\prime}$ is the right derivative of $u$. This simple statement leads to the idea of considering spaces of entire functions more general than weighted Hilbert spaces with integral norm, using the sequence of monomial norms as a research tool. In this case, it is natural to assume the fulfillment of one more property for the space. Let us assume that the space $H$ contains the system of monomials $z^{n}, n \in \mathbb{N} \cup\{0\}$, and this system is complete in $H$. The beginning of the implementation of this idea was laid in [10]. In [11], the authors proved a sufficient condition for the existence of unconditional bases of reproducing kernels.

Theorem A. [11] Let the system of monomials $\left\{z^{n}, n \in \mathbb{N} \cup\{0\}\right\}$ be complete in a radial functional Hilbert space $H$ stable with respect to dividing. If the convex sequence $u(n)=\ln \left\|z^{n}\right\|$ satisfies the condition

$$
\sup _{p \in \mathbb{N}} \inf _{n \in \mathbb{N} \cup\{0\}}\left(u_{+}^{\prime}(n+p)-u_{+}^{\prime}(n)\right)>0,
$$

then the space $H$ possesses unconditional bases of reproducing kernels.

Taking into account the results presented in [6], we assume that for some regularity of the sequence of norms of monomials, condition (2) is very close to the necessary one.

In this paper, we intend to formulate three conditions for the sequence of monomial norms, each of which is equivalent to condition (2).

\section{Sufficient condition in terms of matrix norms.}

Theorem 1. Let $u_{n, k}=u(n)-u(k)-(u(n)-u(n-1))(n-k)$. If $\mathcal{U}$ is a matrix with elements $e^{2 u_{n, k}}, n, k \in \mathbb{N} \cup\{0\}$, then condition (2) is equivalent to

$$
\|\mathcal{U}\|:=\sup _{n}\left(\sum_{k} e^{2 u_{n, k}}\right)^{\frac{1}{2}}<\infty .
$$

Proof. Let us prove that condition (2) implies condition (3). Condition (2) means that for some $p \in \mathbb{N}$ and $\delta>0$ we have

$$
u_{+}^{\prime}(n+p)-u_{+}^{\prime}(n) \geqslant \sigma, n \in \mathbb{N} \cup\{0\} .
$$

Take $n \in \mathbb{N} \cup\{0\}$ and let $k>n$. For a piece-wise linear function $u$ : $u_{+}^{\prime}(n)=u(n+1)-u(n)$. Then

$$
u(k)-u(n)=\sum_{j=0}^{k-n-1}(u(n+j+1)-u(n+j))=\sum_{j=0}^{k-n-1} u_{+}^{\prime}(n+j) .
$$


Hence,

$$
u_{n, k}=\sum_{j=0}^{k-n-1}\left(u_{+}^{\prime}(n-1)-u_{+}^{\prime}(n+j)\right) .
$$

By condition (4),

$$
u_{+}^{\prime}(n+j) \geqslant u_{+}^{\prime}(n-1)+\left[\frac{j}{p}\right] \sigma, \quad n=1,2, \ldots, \quad j=0,1, \ldots,
$$

where $[x]$ denotes the integer part of $x$. Hence,

$$
u_{n, k} \leqslant-\sigma \sum_{j=0}^{k-n-1}\left[\frac{j}{p}\right] \leqslant-\frac{\sigma p}{2}\left(\left[\frac{k-n-1}{p}\right]-1\right)\left[\frac{k-n-1}{p}\right] .
$$

Thus,

$$
\sum_{k=n+1}^{\infty} e^{2 u_{n, k}} \leqslant \sum_{j=0}^{\infty} \exp \left(-\sigma p\left(\left[\frac{j}{p}\right]-1\right)\left[\frac{j}{p}\right]\right):=C(\sigma, p), n \in \mathbb{N} .
$$

Let $k<n$; then

$$
\begin{gathered}
u_{n, k}=u(n)-u(k)-u_{+}^{\prime}(n-1)(n-k)= \\
=\sum_{j=1}^{n-k}\left(u(n-j+1)-u(n-j)-u_{+}^{\prime}(n-1)\right)=\sum_{j=1}^{n-k}\left(u_{+}^{\prime}(n-j)-u_{+}^{\prime}(n-1)\right) .
\end{gathered}
$$

Since $j=s p+1, \ldots,(s+1) p, s=0,1,2, \ldots$, the inequality holds:

$$
u_{+}^{\prime}(n-j)-u_{+}^{\prime}(n-1) \leqslant-s \sigma .
$$

Then

$$
u_{n, k} \leqslant-\frac{p \sigma}{2}\left[\frac{n-k}{p}\right]\left(\left[\frac{n-k}{p}\right]-1\right), k<n .
$$

Hence,

$$
\sum_{k=0}^{n-1} e^{2 u_{n, k}} \leqslant \sum_{j=0}^{\infty} \exp \left(-p \sigma\left[\frac{j}{p}\right]\left(\left[\frac{j}{p}\right]-1\right)\right)=C(\sigma, p), n \in \mathbb{N} .
$$

By (6), this implies condition (3). 
Conversely, let us prove that condition (3) implies condition (2). Assume that (3) holds, but (2) does not. Then, for every $\varepsilon>0$ and for every $p \in \mathbb{N}$, there exists a sequence of natural numbers $n_{m} \rightarrow \infty$, such that

$$
u_{+}^{\prime}\left(n_{m}-1+p\right)-u_{+}^{\prime}\left(n_{m}-1\right) \leqslant \varepsilon, m \in \mathbb{N} .
$$

By (5), for $n=n_{m}, n_{m}<k \leqslant n_{m}+p$ we have

$$
u_{n_{m}, k}=\sum_{j=0}^{k-n_{m}-1}\left(u_{+}^{\prime}\left(n_{m}-1\right)-u_{+}^{\prime}\left(n_{m}+j\right)\right) .
$$

Hence, since $u_{+}^{\prime}$ is nondecreasing,

$$
u_{n_{m}, k} \geqslant-\left(k-n_{m}\right) \varepsilon, \quad k-n_{m}=1, \ldots, p,
$$

and, therefore,

$$
\sum_{k=0}^{\infty} e^{2 u_{n_{m}, k}} \geqslant \sum_{k=n_{m}+1}^{n_{m}+p} e^{-2\left(k-n_{m}\right) \varepsilon}=\sum_{j=1}^{p} e^{-2 j \varepsilon} .
$$

Then for every $\varepsilon>0$ and for every $p \in \mathbb{N}$ we have

$$
\sup _{n \in \mathbb{N} \cup\{0\}} \sum_{k=0}^{\infty} e^{2 u_{n, k}} \geqslant \sum_{j=1}^{p} e^{-2 j \varepsilon},
$$

Hence, $\|\mathcal{U}\|=\infty$, which contradicts to (3).

By Theorem 1, Theorem A can be reformulated as

Theorem A1. If the system of monomials $\left\{z^{n}, n \in \mathbb{N} \cup\{0\}\right\}$ is complete in a radial functional Hilbert space $H$, stable with respect to dividing, and for $u(n)=\ln \left\|z^{n}\right\|, u_{n, k}=u(n)-u(k)-(u(n)-u(n-1))(n-k)$ the matrix $\mathcal{U}$ with elements $e^{2 u_{n, k}}, n, k \in \mathbb{N} \cup\{0\}$, satisfies condition (3), then the space $H$ possesses unconditional bases of reproducing kernels.

3. Sufficient condition in terms of conjugate function and geometric characteristic. Let $\left\{u_{n}\right\}_{n=0}^{\infty}, u_{0}=0$, be a convex increasing sequence of nonnegative numbers. And let $u(t), t \geqslant 0$, be a piece-wise linear function with integer nonnegative breakpoints, $u(n)=u_{n}$. By $\widetilde{u}$ we denote the Young conjugate function of $u$ :

$$
\widetilde{u}(x)=\sup _{t>0}(x t-u(t)), x \in \mathbb{R} .
$$


It is easy to see that the Young conjugate function $\widetilde{u}$ is also piece-wise linear convex, which is equal to 0 as $x \leqslant 0$, and the derivative $\widetilde{u}_{+}^{\prime}$ is a piece-wise constant nondecreasing function with unit jumps at the points $u_{+}^{\prime}(k), k \in \mathbb{N}$.

Theorem 2. Condition (2) is equivalent to the following condition:

$$
\sup _{x \in \mathbb{R}_{+}}\left(\widetilde{u}_{+}^{\prime}(x+d)-\widetilde{u}_{+}^{\prime}(x)\right) \leqslant 2 p+\frac{p d}{\sigma}, d>0
$$

for some $\sigma>0$ and some $p \in \mathbb{N}$.

Proof. Let condition (2) hold. Then we have (4). Take $d>0$. If an interval $[x, x+d)$ does not contain the points $u_{+}^{\prime}(k), k \in \mathbb{N}$, then

$$
\widetilde{u}_{+}^{\prime}(x+d)-\widetilde{u}_{+}^{\prime}(x)=0,
$$

and (7) holds. Otherwise, let

$$
\begin{aligned}
& k_{+}:=\max \left\{k \in \mathbb{N}: \quad u_{+}^{\prime}(k) \in[x, x+d)\right\}, \\
& k_{-}:=\min \left\{k \in \mathbb{N}: u_{+}^{\prime}(k) \in[x, x+d)\right\} .
\end{aligned}
$$

Then

$$
k_{+}-k_{-}+1=\widetilde{u}_{+}^{\prime}(x+d)-\widetilde{u}_{+}^{\prime}(x) .
$$

Let $k_{+}-k_{-}+1 \geqslant 2 p$ and $s=\left[\frac{k_{+}-k_{-}+1}{p}\right]$. Then $s \geqslant 2$. Obviously,

$$
\begin{gathered}
d \geqslant u_{+}^{\prime}\left(k_{+}\right)-u_{+}^{\prime}\left(k_{-}\right) \geqslant \\
\geqslant \sum_{j=0}^{s-2}\left(u_{+}^{\prime}\left(k_{-}+(j+1) p\right)-u_{+}^{\prime}\left(k_{-}+j p\right)\right) \geqslant \sigma(s-1),
\end{gathered}
$$

that is $s \leqslant \frac{d}{\sigma}+1$ and $\widetilde{u}_{+}^{\prime}(x+d)-\widetilde{u}_{+}^{\prime}(x) \leqslant 2 p+\frac{p d}{\sigma}$.

Conversely, let us prove that condition (7) implies condition (2). Let (7) hold, but (2) do not hold. This means that there is a sequence of natural numbers $n_{j} \nearrow \infty$, such that

$$
u_{+}^{\prime}\left(n_{j}+j\right)-u_{+}^{\prime}\left(n_{j}\right)<\frac{1}{j} .
$$

Then, for the unbounded sequence $x_{j}=u_{+}^{\prime}\left(n_{j}\right)$, we have

$$
\widetilde{u}_{+}^{\prime}\left(x_{j}+1\right)-\widetilde{u}_{+}^{\prime}\left(x_{j}\right)>j,
$$


and we obtain a contradiction.

Condition (7) can be written more compactly.

Theorem $\mathbf{2}^{\prime}$. Condition (7) is equivalent to

$$
\sup _{x \in \mathbb{R}_{+}}\left(\widetilde{u}_{+}^{\prime}(x+1)-\widetilde{u}_{+}^{\prime}(x)\right)<\infty .
$$

Proof. Obviously, condition (7) implies condition (8). If (8) holds, then, by monotonicity of $u_{+}^{\prime}$, we have

$$
\sup _{x \in \mathbb{R}_{+}}\left(\widetilde{u}_{+}^{\prime}(x+d)-\widetilde{u}_{+}^{\prime}(x)\right):=M<\infty, d \in(0,1] .
$$

Therefore, for every $d>0$ we have

$$
\sup _{x \in \mathbb{R}_{+}}\left(\widetilde{u}_{+}^{\prime}(x+d)-\widetilde{u}_{+}^{\prime}(x)\right) \leqslant M+M d,
$$

which implies (7).

Let $v$ be a convex function on $\mathbb{R}$, and $p$ be a positive number. Let

$$
\rho(v, x, p)=\sup \left\{t>0: \int_{x-t}^{x+t}\left|v_{+}^{\prime}(\tau)-v_{+}^{\prime}(x)\right| d \tau \leqslant p\right\}
$$

where $v_{+}^{\prime}$ is the right derivative of $v$. This characteristics was introduced in [12].

Lemma 1. For the convex piece-wise linear function $u(t), t \in \mathbb{R}$, condition (8) is equivalent to

$$
\inf _{x>1} \rho(\widetilde{u}, x, 1)>0 .
$$

Proof. Let (8) hold. Then $\sup _{x \in \mathbb{R}_{+}}\left(\widetilde{u}_{+}^{\prime}(x+1)-\widetilde{u}_{+}^{\prime}(x)\right):=N<\infty$. Without loss of generality, we can suppose that $N \geqslant 1$. The monotonicity of the function $\widetilde{u}_{+}^{\prime}(x)$ implies

$$
\int_{x-\frac{1}{2 N}}^{x+\frac{1}{2 N}}\left|\widetilde{u}_{+}^{\prime}(\tau)-\widetilde{u}_{+}^{\prime}(x)\right| d \tau \leqslant 1 .
$$


By definition of $\rho(\widetilde{u}, x, 1)$, this means that $\rho(\widetilde{u}, x, 1) \geqslant \frac{1}{2 N}, x \geqslant 1$. Thus, (9) holds.

Conversely, let $\rho(\widetilde{u}, x, 1) \geqslant 2 \delta>0, x \geqslant 1$. By definition of $\rho(\widetilde{u}, x, 1)$, we have

$$
\int_{\delta}^{2 \delta}\left(\widetilde{u}_{+}^{\prime}(x+t)-\widetilde{u}_{+}^{\prime}(x)\right) d t \leqslant 1, x \geqslant 1,
$$

and, therefore, $\widetilde{u}_{+}^{\prime}(x+\delta)-\widetilde{u}_{+}^{\prime}(x) \leqslant \frac{1}{\delta}, x \geqslant 1$. Let $N=\left[\frac{1}{\delta}\right]+1$. Taking into account that $\widetilde{u}_{+}^{\prime}$ is an increasing function, we get:

$\widetilde{u}_{+}^{\prime}(x+1)-\widetilde{u}_{+}^{\prime}(x) \leqslant \sum_{k=0}^{N-1}\left(\widetilde{u}_{+}^{\prime}(x+k \delta+\delta)-\widetilde{u}_{+}^{\prime}(x+\delta)\right) \leqslant \frac{N}{\delta} \leqslant N^{2}, x>0$, that is, (8) holds.

Lemma 2. Condition (9) is equivalent to

$$
\sup _{t>0} \rho(u, t, 1)<\infty .
$$

Proof. Let $\rho(u, t, 1) \leqslant N, t \in \mathbb{R}_{+}$, for some constant $N>0$. Without loss of generality, we can suppose that $N$ is integer. By definition of $\rho(u, t, 1)$, this means that

$$
\int_{t-N}^{t+N}\left|u_{+}^{\prime}(y)-u_{+}^{\prime}(t)\right| d y \geqslant 1, t \in \mathbb{R}_{+} .
$$

Hence, since $u_{+}^{\prime}(y)$ is a monotonic function, we have

$$
u_{+}^{\prime}(n+N)-u_{+}^{\prime}(n-N) \geqslant \frac{1}{2 N}, n \in \mathbb{N},
$$

or

$$
u_{+}^{\prime}(n+2 N)-u_{+}^{\prime}(n) \geqslant \frac{1}{2 N}, n \in \mathbb{N} .
$$

As we noted at the beginning of this section, the function $\widetilde{u}$ is piecewise linear with breakpoints $x_{n}=u_{+}^{\prime}(n-1)=u(n)-u(n-1)$, and the derivative $\widetilde{u}_{+}^{\prime}$ has unit jumps at the points $x_{n}$. Thus, the last estimate can be written as

$$
x_{n+2 N}-x_{n} \geqslant \frac{1}{2 N}, n \in \mathbb{N} .
$$


This means that the quantity of jumps of $\widetilde{u}_{+}^{\prime}$ on an interval of length less than $\frac{1}{2 N}$ does not exceed $2 N$. Since there are unit jumps, we find that for $\varepsilon<\frac{1}{2 N}$

$$
\widetilde{u}_{+}^{\prime}(x+\varepsilon)-\widetilde{u}_{+}^{\prime}(x) \leqslant 2 N, x \geqslant 1 .
$$

Put $\varepsilon=\frac{1}{5 N}$. Then

$$
\int_{t-\varepsilon}^{t+\varepsilon}\left|\widetilde{u}_{+}^{\prime}(x)-\widetilde{u}_{+}^{\prime}(t)\right| d x \leqslant 2 N \cdot 2 \varepsilon=\frac{4}{5}<1, t \geqslant 1 .
$$

Hence,

$$
\rho(\widetilde{u}, t, 1) \geqslant \frac{1}{5 N}, t \geqslant 1
$$

Conversely, let

$$
\rho(\widetilde{u}, t, 1) \geqslant 2 \varepsilon, t \geqslant 1
$$

for some $\varepsilon>0$. Then

$$
\int_{x+\varepsilon}^{x+2 \varepsilon}\left|\widetilde{u}_{+}^{\prime}(y)-\widetilde{u}_{+}^{\prime}(x)\right| d y \leqslant \int_{x-2 \varepsilon}^{x+2 \varepsilon}\left|\widetilde{u}_{+}^{\prime}(y)-\widetilde{u}_{+}^{\prime}(x)\right| d y \leqslant 1 .
$$

Hence, for any $x \geqslant 1$,

$$
\widetilde{u}_{+}^{\prime}(x+\varepsilon)-\widetilde{u}_{+}^{\prime}(x) \leqslant \frac{1}{\varepsilon} .
$$

Put $N=\left[\frac{1}{\varepsilon}\right]$. Then $\widetilde{u}_{+}^{\prime}(x+\varepsilon)-\widetilde{u}_{+}^{\prime}(x) \leqslant N+1$, or

$$
u_{+}^{\prime}(n+N+1)-u_{+}^{\prime}(n) \geqslant \varepsilon, n \in \mathbb{N} \cup\{0\} .
$$

Thus,

$$
\int_{n+N+1}^{n+2(N+1)}\left|u_{+}^{\prime}(x)-u_{+}^{\prime}(n)\right| d x \geqslant \varepsilon(N+1)>1 .
$$

Hence, $\rho(u, n, 1) \leqslant 2 N+2$. It was proved in [13] (see Lemmas 3 and 4) that the function $\rho(u, x, 1)$ satisfies the Lipschitz condition

$$
|\rho(u, x, 1)-\rho(u, y, 1)| \leqslant|x-y|, x, y \in \mathbb{R} .
$$


Therefore, $\rho(u, t, 1) \leqslant 2 N+3, t \in \mathbb{R}_{+}$. $\square$

In conclusion, we present a theorem that follows from the results of [10]. Theorem 3. Let $H$ be a radial functional Hilbert space stable with respect to dividing, in which the system of monomials is complete. If one of conditions (2), (3), (8), (9) or (10) holds, then

1. the space $H$ as a Banach space is isomorphic to the space of entire functions with the norm

$$
\|F\|^{2}=\frac{1}{2 \pi} \int_{0}^{\infty} \int_{0}^{2 \pi}\left|F\left(r e^{i \varphi}\right)\right|^{2} e^{-2 \widetilde{u}(\ln r)} d \varphi d \widetilde{u}_{+}^{\prime}(\ln r) ;
$$

2. the Bergman function of the space $H$ satisfies the condition

$$
K(z) \asymp e^{2 \widetilde{u}(\ln |z|)}, z \in \mathbb{C} .
$$

\section{Proof.}

1. By Theorem 4 in [10], if condition (3) is satisfied, then the space $H$ as a Banach space is isomorphic to the space of entire functions with the norm

$$
\|F\|_{0}^{2}:=\frac{1}{2 \pi} \sum_{n=1}^{\infty} e^{-2 \widetilde{u}\left(\ln R_{n}\right)} \int_{0}^{2 \pi}\left|F\left(R_{n} e^{i \varphi}\right)\right|^{2} d \varphi,
$$

where $R_{n}=e^{u_{+}^{\prime}(n-1)}$. By Lemma 2 in [10], $\widetilde{u}_{+}^{\prime}(\ln r)$ is a piece-wise constant nondecreasing function with unit jumps at the points $R_{n}$. Therefore, norm (13) coincides with norm (11).

2. By Theorem 3 in [10], if condition (3) is satisfied, then (12) is true.

Acknowledgments. The research was supported by the grant of Russian Science Foundation (project no. 21-11-00168).

\section{References}

[1] Seip K. Density theorems for sampling and interpolation in the BargmannFock space I. Reine Angew. Math., 1992, vol. 429, pp. 91-106.

DOI: http://doi.org/10.1515/crll.1992.429.91

[2] Seip K., Wallsten R. Density theorems for sampling and interpolation in the Bargmann-Fock space II. Reine Angew. Math., 1992, vol. 429, pp. 107-113. http://eudml.org/doc/153434 
[3] Borichev A., Dhuez R., Kellay K. Sampling and interpolation in large Bergman and Fock spaces. Journal of Functional Analysis, 2007, vol. 242, no. 2, pp. 563-606. DOI: http://doi.org/10.1016/j.jfa.2006.09.002

[4] Borichev A., Lyubarskii Yu. Riesz bases of reproducing kernels in Fock type spaces. Journal of the Institute of Mathematics of Jussieu, 2010, vol. 9, pp. 449-461. DOI: http://doi.org/10.1017/S147474800900019X

[5] Baranov A., Belov Yu., Borichev A. Fock type spaces with Riesz bases of reproducing kernels and de Branges spaces. Studia Mathematica, 2017, vol. 236 , no. 2 , pp. 127-142.

DOI: http://doi.org/10.4064/sm8504-9-2016

[6] Isaev K. P., Yulmukhametov R. S. Unconditional bases of reproducing kernels in Hilbert spaces of entire functions. Ufa Math. J., 2013, vol. 5, no. 3, pp. 67-76. DOI: http://doi.org/10.13108/2013-5-3-67

[7] Isaev K. P., Lutsenko A. V., Yulmukhametov R. S. Unconditional bases in weakly weighted spaces of entire functions. St. Petersburg Math. J., 2019, vol. 30, no. 2, pp. 253-265. DOI: http://doi.org/10.1090/spmj/1541

[8] Isaev K. P., Trunov K. V., Yulmukhametov R. S. Equivalent Norms in Hilbert Spaces with Unconditional Bases of Reproducing Kernels. Journal of Mathematical Sciences, 2020, vol. 250, pp. 310-321.

DOI: http://doi.org/10.1007/s10958-020-05017-3

[9] Isaev K. P., Yulmukhametov R. S. Unconditional bases in radial Hilbert spaces. Vladikavkaz. Matem. Zhurn., 2020, vol. 22, no. 3, pp. 85-99. (in Russian). DOI: http://doi.org/10.46698/q8093-7554-9905-q

[10] Isaev K. P., Yulmukhametov R. S. Geometry of radial Hilbert spaces with unconditional bases of reproducing kernels. Ufa Math. J., 2020, vol. 12, no. 4, pp. 55-63. DOI: http://doi.org/10.13108/2020-12-4-55

[11] Isaev K. P., Yulmukhametov R. S. On a sufficient condition for the existence of unconditional bases of reproducing kernels in Hilbert spaces of entire functions. Lobachevskii Journal of Mathematics, 2021, vol. 42, no. 6, pp. 1154-1165. DOI: http://doi.org/10.1134/S1995080221060093

[12] Lutsenko V. I., Yulmukhametov R. S. Generalization of the Paley-Wiener theorem in weighted spaces. Math. Notes, 1990, vol. 48, no. 5, pp. 11311136. DOI: http://doi.org/10.1007/BF01236300

[13] Bashmakov R. A., Isaev K. P., Yulmukhametov R. S. On geometric characteristics of convex functions and Laplace integrals, Ufimskij Matem. Zhurn., 2010, vol. 2, no. 1, pp. 3-16 (in Russian).

http://mathnet.ru/eng/ufa/v2/i1/p3 
Received May 24, 2021.

In revised form, October 4, 2021.

Accepted October 5, 2021.

Published online October 22, 2021.

Institute of Mathematics, Ufa Federal Research Centre,

Russian Academy of Sciences (Institute of Mathematics UFRC RAS)

112, Chernyshevsky str., Ufa, Russia, 450008

E-mail: orbit81@list.ru,yulmukhametov@mail.ru 